\title{
A Hybrid Pattern Recognition System for Detecting Buried Object in GPR Images
}

\author{
${ }^{* 1}$ U. Özkaya, ${ }^{2}$ L. Seyfi \\ ${ }^{1,2}$ Department of Electrical and Electronics Engineering, Selcuk University, Konya, 42075, Turkey \\ E-mail: ${ }^{1}$ uozkaya@selcuk.edu.tr, leventseyfi@ selcuk.edu.tr
}

Received: 20 $^{\text {th }}$ December 2017, Accepted: 20th January 2018, Published: 28th February 2018

\begin{abstract}
It is very difficult to evaluate the shape of the buried objects using Ground Penetrating Radar (GPR). In this study, 180 GPR B scan images were classified by using different classification algorithms. GPR-B scan images of objects with different shapes in various depths were obtained by using GprMax simulation program. Noise in these images were eliminated by Wavelet transform and then, Gray Level Co-occurrence Matrices (GLCM), run length matrix (RLM) and Autocorrelation function (ACF) features were extracted from the segmented images Some feature reduction methods, which are Principle Component Analysis and Independent Component Analysis, provided that size of the feature vectors was decreased. All the modified vectors were given as inputs to various classifiers. Outputs of the classifier systems were evaluated and compared with each other. Accuracy values of proposed algorithm results were computed. Consequently, it is possible to use in real application of GPR devices.
\end{abstract}

\section{Introduction}

Ground Penetrating Radar (GPR) is a type of radar, provides information for many subsurface investigation including buried object detection, preliminary research for archaeological excavation, inverse scattering, and tomographic imaging. Besides, GPR is one of the non-destructive methods which is preferred for oil reserves and natural gas exploration, conduit and pipe localization. Sensors or antenna arrays can be used for underground imaging depending on the variety of applications. The GPR imaging technique is based on the transmitting and receiving of the electromagnetic wave in the field at a few meters depth with specific steps at the centimeter level. In fact, GPR uses wave reflections in regions where dielectric discontinuities exist in underground. Dielectric discontinuities can be formed by different soil layers, buried objects or clutter. The acquisition of information about the relevant region is per-formed depending on the time delay and phase calculation of the radar pulse. Neglecting wave refraction on the ground surface during inverse calculations of circular wave propagation can lead to incorrect configuration of the GPR images and incorrect detection of the target position.
GPR B scan images consist of a combination of 1-D GPR A scan signals. GPR response is defined as combination of attenuation level in each depth index at measurement point. This response is called as 1D GPR A scan signal. 2-D GPR B scan image is obtained by joining the received GPR A scan signal side by side in a specific step sequence. 3-D GPR C scan image is obtained by using 2-D GPR B scan images. In GPR B scan images, buried objects appear as hyperbola. The types of hyperbola differs in accordance with the material, shape and depth of buried objects.

The literature consists of few work related to detection and classification of buried objects patterns. Shihab et al. [1] used curve fitting method on the hyperboles in the GPR B scan image to determine the radius of buried cylindrical objects. Capineri et al. [2] applied Hough transform to segment linear lines owing to electrical discontinuity and determined the location of the buried objects. Caorsi et al. [3] estimated the properties of the buried cylinder material using artificial neural networks. Dolgiy et al. [4] used to determined radius of buried object via the weighted least squares method, the recursive Kalman filter technique and the maximum likelihood method. A scan signals, obtained from the reflected waves due to the dielectric difference, were separated into different frequency components to detect the existence of the object underground plane [5]. Delbo et al. [6] proposed a cascade system consisting of wavelet transform and fuzzy classification methods. Thanks to wavelet transform, the noise in the GPR B scan image is reduced and then, the hyperbolas in the image are segmented by fuzzy $\mathrm{k}$ means algorithm. Preprocessing algorithms were applied to reduce the noises around hyperbolas in GPR images by Gamba et al. Afterwards, GPR images were interpreted by artificial neural networks and detector design was performed [7]. Al-Nuaimy et al. [8] performed preprocessing algorithms to reduce undesired noise on the image. Artificial neural network was successful for segmentation of GPR images. At the end, Hough transformation is applied to identify hyperbolas. Effects of different noise levels on GPR images were analyzed over detection of embedded objects by using artificial neural networks [9]. Rossini et al. [10] proposed an interpolation based wavelet transform method for the detection of underground objects. The high-level statistical 
properties obtained from the GPR B scan images are inputs of the artificial neural network and the earth surface and buried object are distinguished in the output layer [11]. Gamba et al. [12] have proposed two different methods, namely template matching and artificial neural network, for the detection of underground objects in small dimensions. Falorini et al. [13] eliminated found noise on GPR images by means of preprocessing algorithms and then, determined the same phase polarity point using 1-D gradient filter. In this way, the location of the target objects were detected. Dell'Acqua et al. [14] implemented 3-D Radon transformations on GPR B scan images to remove buried fake targets. Gurbuz et al. [15] performed the detection of the liner patterns on the GPR images by 2-D radon transformation. Borgioli et al. [16] estimated the location of the buried object by modifying the Hough transformation with weight factors. Pasolli et al. [17] classified hyperbolic and linear patterns with support vector machines by formulating them with genetic optimization descriptor.

This study is divided into three categories in terms of stages. Initially, noise on the GPR B scan images are diminished with wavelet transform and the images are segmented by the Otsu thresholding method. Hough transform is used to separate the buried object and the ground plane. GLCM, RLM and ACF features are extracted from the segmented images. Thanks to various classification algorithms, the shape of the buried objects is estimated. The remaining parts of this paper is organized as follows. The image processing and feature extraction methodologies are described in Section II. Feature reduction and classification methods are introduced in Section III. The experimental results are summarized in Section IV. Conclusions are existed in Section V.

\section{Material and Methods}

In the proposed methodology, firstly buried objects in the GPR image should be detected. As mentioned before, it is necessary for reduction of the noise by preprocessing method. Also, image segmentation is required for sensing of buried objects.

\subsection{Preprocessing and Segmentation}

The GPR device is exposed to electromagnetic noise and interference, which may cause deterioration of the scanned image. Pre-processing algorithms are used to reduce noise on images, remove undesired surface echo, and compensate for propagation losses. It is possible to distinguish high frequency noise components in images by using 2-D Wavelet transform. The simplest approach in 2-D Wavelet transform is to transform a 1-D wavelet into a 2-D Wavelet by tensor multiplying. As a result of this operation, 4 different wavelet functions are obtained as Scaling function $(\Phi)$, Horizontal wavelet $\left(\Psi^{\mathrm{H}}\right)$, Vertical wavelet $\left(\Psi^{\mathrm{V}}\right)$ and Diagonal wavelet $\left(\Psi^{\mathrm{D}}\right)$.
Wavelet models are expressed by Eq. (1), Eq. (2), Eq. (3) and Eq. (4).

$$
\begin{gathered}
\Phi(x, y)=\Phi(x) \cdot \Phi(y) \\
\Psi^{H}(x, y)=\Psi(x) \cdot \Phi(y) \\
\Psi^{V}(x, y)=\Phi(x) \cdot \Psi(y) \\
\Psi^{D}(x, y)=\Psi(x) . \Psi(y)
\end{gathered}
$$

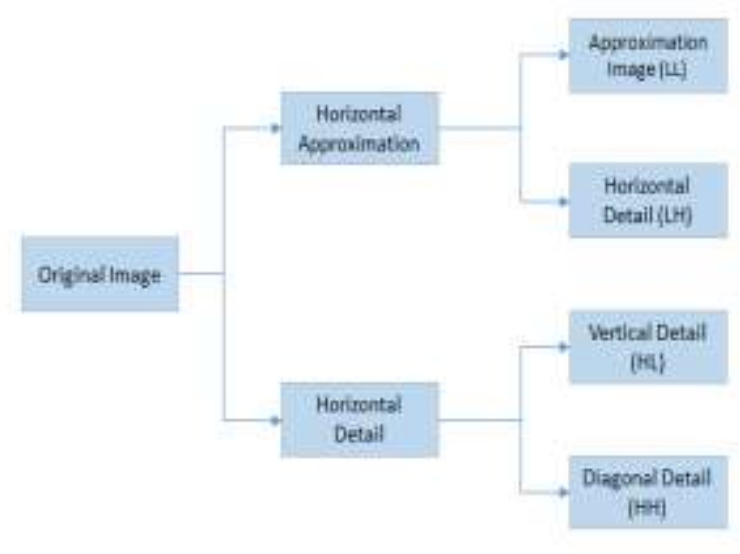

Fig. 1. Block Diagram of 2-D Discrete Wavelet Transform

Intensity level changes in 2-D images and gray level changes in different orientations can be measured by using these wavelet functions. In the 2-D Wavelet transform, the image components of the signal plane are divided into 4 sub-bands as LL (Approximation Image), LH (Horizontal Detail), HL (Vertical Detail) and HH (Diagonal Detail). Basic idea of 2-D Discrete Wavelet transform is shown Fig. 1. If the level of decomposition is to be increased, more wavelet transforms are applied to LL again

The pre-processed image is passed through two more steps for segmentation. In order to segment buried objects with specific dielectric property and ground plane in the reconstructed image, Otsu thresholding method is applied. This distinguishable process, which allows to light up parts of an image containing potential targets, is based on the assumption that buried objects are often associated with large amplitude echoes. It is the thresholding method based on the analysis of the global image histogram and on the average of the entropy of the "object" and "background" classes. Pixels are divided into two classes as $C_{0}$ and $C_{l}$, according to the previously determined gray level $(t)$. Taking into account the gray level distribution probability, $C_{0}$ and $C_{l}$ are calculated by using Eq. (5) and Eq. (6)

$$
\omega_{0}=P\left(C_{0}\right)=\sum_{i=0}^{t-1} P_{i}
$$




$$
\omega_{1}=P\left(C_{1}\right)=\sum_{i=t}^{L-1} P_{i}
$$

The means of $C_{0}$ and $C_{l}$ classes are computed as Eq. (7) and Eq. (8).

$$
\begin{aligned}
& \mu_{0}=\sum_{i=0}^{t-1} i . P_{i} / \omega_{0} \\
& \mu_{1}=\sum_{i=t}^{L-1} i . P_{i} / \omega_{1}
\end{aligned}
$$
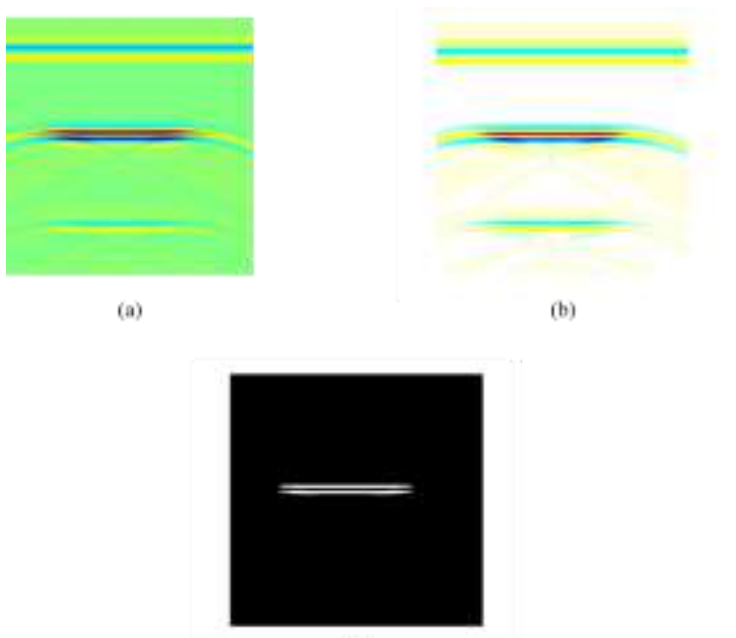

Fig. 2. a) Original GPR Image b) Wavelet transform applied GPR Image c) Segmented GPR Image (9).

The total mean of all classes is expressed by Eq.

$$
\mu_{T}=\omega_{0} \mu_{0}+\omega_{1} \mu_{1}
$$

The variances of $C_{0}$ and $C_{l}$ classes are calculated as Eq. (10) and Eq. (11).

$$
\begin{aligned}
\sigma_{0}^{2} & =\sum_{i=0}^{t-1}\left(i-\mu_{0}\right)^{2} p_{i} / \omega_{0} \\
\sigma_{1}^{2} & =\sum_{i=t}^{L-1}\left(i-\mu_{1}\right)^{2} p_{i} / \omega_{1}
\end{aligned}
$$

The within-class variance is computed as in Eq. (12).

$$
\begin{gathered}
\sigma_{\omega}^{2}=\sum_{k=t}^{M} \omega_{k} \sigma_{k}^{2} \\
\sigma_{\omega}^{2}=\sum_{k=t}^{M} \omega_{k} \sigma_{k}^{2}
\end{gathered}
$$

In Otsu method, between-class variance is minimized when the within-class variance is maximized. The between-class variance is formulated as in Eq. (13).

$$
\sigma_{B}^{2}=\left(\omega_{0}\right)\left(\mu_{0}-\mu_{T}\right)^{2}+\left(\omega_{1}\right)\left(\mu_{1}-\mu_{T}\right)^{2}
$$

The Hough transformation is based on the principle of coordinate transformation in object recognition for digital image analysis. This transformation is aimed to reveal the shape of desired object using cumulative distribution procedure. In addition, Hough transform is an image analysis method used to detect linear or non-linear shapes [18]. Shapes in 2-D x-y coordinates system are converted to a space which consists of rotation angle $(\theta)$ and scaling factor (s) parameters [19]. Buried objects in GPR images show nonlinear characteristics. In the segmented images, Hough transform is applied to distinguish between ground plane and buried object. Briefly, high frequency noise in GPR images was decreased by Wavelet transform. Then, pre-processed GPR images were segmented by Otsu thresholding method. Finally, Hough transformation was used to eliminate the ground plane in segmented GPR images. All procedure results are shown in Fig. 2.

Feature Extraction and Reduction

Feature extraction can be defined as a special type of dimensionality reduction technique [20]. In addition to feature extraction, the size of the feature vector may need to be changed. In order to perform classification algorithm, feature extraction is necessary for detection of buried objects. For such purpose, Gray Level Co-occurrence Matrices (GLCM) Features were obtained from segmented GPR B Scan images. The joint probability value in gray level values of some pixel sets is known as GLCM. It is calculated how many times i th pixel occurs jointly with another pixel which has same gray value of $i$ th pixel. Too many GLCM properties can be obtained by changing the displacement vector and direction among such pixel pairs. Four GLCMs with directions $\left(0^{\circ}, 45^{\circ}, 90^{\circ}\right.$ and $\left.135^{\circ}\right)$ are created for each sample image segment at the exact distance. Once the GLCM is normalized, we can derive statistical properties for each sample: autocorrelation, contrast, correlation, cluster prominence, cluster shade, dissimilarity, energy, entropy, homogeneity, maximum probability, sum of squares, sum average, sum variance, sum entropy, difference variance, difference entropy and inverse difference.

The run length matrix (RLM) is defined as the number of pixels in the different directions on the grey level image [21]. Run length matrix is generated for each image for $0^{\circ}, 45^{\circ}, 90^{\circ}$ and $135^{\circ}$. Five statistical features, which are short run emphasis, long run emphasis, grey level nonuniformity, run length non-uniformity and run percentage, are extracted.

Autocorrelation function (ACF) is applied after subtracting the mean. The main purpose is to duplicate certain models in the image. Autocorrelation can be formulated as in Eq. (14).: 


$$
\rho(x, y)=\frac{\sum_{i=1}^{M-x} \sum_{j=1}^{N-y}(I(i, j)-\mu)(I(i+x, j+y)-\mu)}{(M-x)(N-y)}
$$

where $x, y$ are the amount of shifts in grey value in $M x N$ image. $\mu$ is described as mean of image. In $\mathrm{ACF}$, the peaks are located in the horizontal and vertical margins by least squares interpolation. Thus, ACF consists of four different parameters which are the horizontal and vertical margins.

Principle Component Analysis (PCA) is a wellknown method for extracting and resizing of features. It allows the size of the data set containing a large number of interrelated variables to be reduced in lower size while preserving the existing changes in the data as much as possible [22]. This analysis aims to determine the best transformation that can be expressed with fewer variables in the given data. After the transformation, the obtained variables are called as principle components of the first variables. The first principal component variance value is the largest and the other principal components are sorted in order of decreasing variance values. Principle axes $\left(T_{1}, T_{2}, \ldots, T_{m}\right)$ are orthonormal axes for $p$-dimensional data set. PCA basis vectors are described as eigenvectors of the covariance matrix $S$ defined as:

$$
S=\frac{1}{N} \sum_{i=1}^{M}\left(x_{i}-\mu\right) \cdot\left(x_{i}-\mu\right)^{T}
$$

Where $\mu_{r}$ represents the means of data set, $x_{i}$ is $i$ th column vector. $N$ is called as number of samples. In Eq. (16), $\lambda_{i}$ is the largest eigenvalue in $S$ samples.

$$
S T_{i}=\lambda_{i} T_{i}, \quad i \in 1, \ldots, m
$$

$m$ number of independent components are given in Eq. (17).

$$
y=\left[y_{1}, y_{2}, \ldots . ., y_{m}\right]=\left[T_{1}^{T} x, T_{2}^{T}, \ldots ., T_{m}^{T}\right]=T^{T} x
$$

In projection space, $m$ number of principle components are necessary to be decorrelated. The variation of the $S$-data is determined on a global basis and the principal axes are formed from the global covariance matrix.

$$
\hat{S}=\frac{1}{N} \sum_{j=1}^{K} \sum_{i=1}^{N_{j}}\left(x_{j}-\hat{\mu}\right)\left(x_{j}-\hat{\mu}\right)^{T}
$$

where number of classes is symbolized as $K, \hat{\mu}$ is global mean in all $S$ samples and on top of that $N_{j}$ is number of samples in $j$ th class. $m$ leading eigenvector is computed in Eq. (19).

$$
\hat{S} T_{i}=\hat{\lambda}_{i} T_{i}, \quad i \in 1, \ldots, m
$$

where $\hat{\lambda}_{i}$ is the $i$ th largest eigenvalue, number of classes is symbolized as $K, \hat{\mu}$ is global mean in all $S$ samples and on top of that $N_{j}$ is number of samples in $j$ th class. The assumption for feature reduction using PCA is that subspaces are covered by the principle axes [23]. Thus, the original data vectors can be represented by the principal component vector whose size is determined as $m$.

Independent Component Analysis (ICA) can linearly separate the random data set into its subcomponents and provide maximum independence between each other's [24]. At the same time, the characteristics remain the same in the analysis. Basically, the data are transformed into a linear system to separate the independent components. Unlike PCA, ICA has transformation process based on correlation. ICA reduces most of the high-level statistical dependencies rather than separating the various components [25]. ICA produces independent components in a linear manner using $x$ data set $\left(x_{1}(t), x_{2}(t), \ldots \ldots, x_{N}(t)\right)$ in Eq. (20).

$$
\left(\begin{array}{c}
x_{1}(t) \\
x_{2}(t) \\
\cdot \\
\cdot \\
\cdot \\
x_{N}(t)
\end{array}\right)=A\left(\begin{array}{c}
s_{1}(t) \\
s_{2}(t) \\
\cdot \\
\cdot \\
\cdot \\
s_{N}(t)
\end{array}\right)
$$

where $A$ is an random coefficient matrix and made as independent as possible.

$$
u(t)=W x(t)=W A S(t)
$$

where $W$ is linear transformation matrix, $u(t)$ represents as un-mixing data. After feature extraction and reduction process, information about the shape of buried object is inferred via various classifier methods such as Linear Discriminant Analysis (LDA), Quadratic Discriminant Analysis (QDA), k-Nearest Neighbor (KNN), Artificial Neural Network (ANN), Support Vector Machine (SVM) and Decision Trees (DT).

\subsection{Classification Algorithms}

After the feature-extraction step, information about the material of the object is inferred by means of an SVM classifier, which has proved effective in various application fields. A brief discussion of this classifier is illustrated in the next section.

LDA is based on a method of transforming the original real matrix into a better separation space by projection method [26]. If $Y_{i}(i=1,2,3, \ldots \ldots C)$ is considered as a training data, $w$ weight vector of the training data in $D$ dimension can be classified by $W$ weight matrix. The between-class scatter matrix is denoted as $S_{b}$, and the within-class scatter matrices is defined as $S_{w}$.

$$
S_{b}=\sum_{1}^{c} N_{i}\left(\mu_{i}-\mu\right)\left(\mu_{i}-\mu\right)^{T}
$$


Where $N_{i}$ is $i$ th class of patterns, $\mu_{i}$ is the mean of $i$ th class and $\mu$ is the mean of total data set.

$$
S_{w}=\sum_{1}^{c} \sum_{y \in Y_{i}}^{1}\left(y-\mu_{i}\right)\left(y-\mu_{i}\right)^{T}
$$

The between-class distance is maximized while within-class distance of data is minimized as in objective function $J$.

$$
J=\frac{\operatorname{tr}\left(W^{T} S_{b} W\right)}{\operatorname{tr}\left(W^{T} S_{w} W\right)}
$$

In Eq. 24, tr is defined as trace of matrix. Langrage Multiplier method is used for maximizing the objective function in order to train LDA classifier.

QDA is a generalized type of linear discriminant analysis. In QDA, data must be separated into two classes and the data must be distributed normally. QDA assumes that the covariance of each class is the same [27]. $\omega_{i}$ represents the $i$ th class in the $M$-class data set. $x$ is element of $R^{k}$ vector space. The conditional probability of $\omega_{i}$ by $x$ is $p\left(\omega_{i} \mid x\right)$.

$$
\begin{gathered}
p\left(\omega_{i} \mid x\right)>p\left(\omega_{j} \mid x\right) \quad \text { for all } j \neq i \\
p\left(\omega_{i} \mid x\right)=p\left(\omega_{j} \mid x\right) p\left(\omega_{i}\right) / p(x)
\end{gathered}
$$

where $p\left(\omega_{i}\right)$ is the probability of $i$ th class and $p(x)$ is the probability density of $x$ vector for all classes. When the solution is modified by substituting Eq. (25) into Eq. (26):

$$
p\left(x \mid \omega_{i}\right)>p\left(x \mid \omega_{j}\right) \quad \text { for all } j \neq i
$$

In QDA, there is a parabola or hyperbolic conic surface in order to separate subspaces from each other. The discriminant function is formulated as in Eq. (28).

$$
d_{k}(x)=2 \mu_{k}^{T} \sum_{k}^{-1} x-\mu_{k}^{T} \sum_{k}^{-1} \mu_{k}-2 \log \pi_{k}
$$

where $\mu_{k}$ is mean vector, the prior probability is defined as $\pi_{k}$ and covariance matrix is symbolized as $\sum_{k}$. Discriminant rule is given as in Eq. (29). In QDA rule, $p(k / x)$ is posterior distribution.

$$
d_{k}(x)=\min _{1 \leq k \leq K} d_{k}(x) \Leftrightarrow \max _{1 \leq k \leq K} p(k / x)
$$

The KNN algorithm can perform simple and comprehensive classification using neighborhood features. The error probability of the KNN algorithm for any class is greater than for the Bayes classifier [28]. For this reason, it can be generalized that half of the total information required for classification is located in the nearest neighbors. The performance of KNN varies depending on two factors [29]. First of all, it is very important to find a suitable $k$ values for a specific problem. When the class boundaries are determined more precisely at large $k$ values, these values are minimally affected from noise. The values of $k$ can vary depending on classification problem. Another factor on performance of the classification algorithm is distance metric. KNN shows high accuracy in classifying data sets with large dimensions. For small data sets, high computational complexity is required due to the precision in calculating the distance. Therefore, whatever the size of the data, all distances must be calculated in the new data used for classification purposes. In distance metric calculation, Manhattan and Euclidean distance are used in Eq. (30). Also, Mahalanobis distance is computed as in Eq. (31).

$$
\begin{gathered}
L_{m}(p, q)=\left(\sum_{i=1}^{n}\left(\mid p_{i}-q_{i}\right)^{m}\right)^{1 / m} \\
M_{k}(p, q)=\sqrt{\left(p_{i}-q_{i}\right)^{T} \sum_{k}^{-1}\left(p_{i}-q_{i}\right)}
\end{gathered}
$$

ANN is a network structure consisting of nodes and neurons with parallel processing capability [30]. Weights and biases need to be updated for desired network properties in ANN. There are two application stages in ANN such as training and testing parts. In the training phase, feature data is given as input to the network. The network weights and bias are updated according to the characteristics of the input and output layers. Transfer functions are used to reduce the linearity of the network. The training phase can take a long time. Using the network generalization feature in the test phase, it has ability to estimate output response with high accuracy. For this reason, ANN is often used to solve complex optimization and classification problems [31]. One of the most preferred training algorithms in classification problems is the backpropagation (BP) algorithm [32].

SVM bases on statistical learning mentality. It is the supervised learning method used for classification and regression analysis [33]. SVM targets the global minimum in the training phase, depending on error parameter. SVM works on the basis of structural and empirical risk minimization in traditional neural networks. Empirical Risk Minimization (ERM) reduces the training error to the lowest, while Structural Risk Minimization (SRM) reduces the expected maximum risk. Where training data is labelled as $\{x i, y i\}, y_{i} \in\{-1,1\}, x_{i} \in$ $R^{d}$. Some hyperplanes exist to separate negative and positive classes from each other. The $x$ points on the hyperplane provide the equality $w \cdot x+b=0$. Where $w$ can be defined as normal to hyperplane, $b / w$ is the perpendicular distance between hyperplane and origin and Euclidean norm of $w$ represents $\|w\|$. The shortest distances to the positive and negative classes are denoted as $\mathrm{d}_{+}, \mathrm{d}_{\text {., }}$ respectively. The total margin is calculated as $\mathrm{d}_{+}+\mathrm{d}$.. Major headings should be typeset in boldface with the first letter of important words capitalized. SVM aims to obtain the largest margin between positive and negative classes. There are some restrictions in the training phase, as in Eq. (32) and Eq. (33). 


$$
\begin{array}{ll}
x_{i} \cdot w+b \geq+1 & \text { for } y_{i}=+1 \\
x_{i} \cdot w+b \leq-1 & \text { for } y_{i}=-1
\end{array}
$$

where the above equations are modified as in Eq. (34).

$$
y_{i}\left(x_{i} \cdot w+b\right)-1 \geq 0 \quad \forall \mathrm{i}
$$

The set of points for which the equality in Eq. (32) and Eq. (33), these points are on $H_{1}: x_{i} \cdot w+b=1$ and $H_{2}: x_{i} \cdot w+b=-1$. Perpendicular distance to the origin is equal to $1-b /\|w\|$ while $w$ is these hyperplanes. Therefore, $d_{+}=d_{-}=1 \Lambda|w| \mid$ and total margin is $d_{+}+d_{-}=2 \bigwedge \mid w \|$.

When vectors allocate data space to non-linear areas SVM uses kernel functions to transfer data to a different space. Inner product in different feature space are defined as kernel function. Kernel functions increase the data size to provide more optimal vector separation. In high dimensional space transformation $(\Phi: x \rightarrow \varphi(x))$, dot product refers as $K\left(x_{i}, x_{j}\right)=\varphi\left(x_{i}\right)^{T} \varphi\left(x_{j}\right) . K_{i j} \equiv K\left(x_{i}, x_{j}\right)$ is kernel matrix in the Euclidean space. The radial basis function is defined as in Eq. (35).

$$
K\left(x^{t}, x\right)=\exp \left[-\frac{\left\|x^{t}-x\right\|^{2}}{\sigma^{2}}\right]
$$

where $x^{t}$ is the center of kernel function and $\sigma$ is radius of kernel.

$\mathrm{T}$ is learning-based algorithm. This algorithm iteratively evaluates the data. DT consists of one root node and many internal nodes. Each of the nodes has a labelled feature. For this reason, nodes in the decision tree are necessary to be updated in each iteration. DT heuristically uses entropy or knowledge gain in feature selection [34]. It is aimed at obtaining the best feature in the selection process. After completion of the learning process, links between nodes in the decision tree can be seen. On this count, insignificant features in the data set are reached. It also has the ability to automatically evaluate features after learning stage [35].

\section{Proposed Algorithm}

The proposed method for detecting buried objects consists of 4 different stages. These are preprocessing, feature extraction, feature reduction and classification process respectively. GPR image in Fig. 3 contains multiple buried objects. Numerous objects can be sensed due to the overlap of the hyperbolas represented by these objects. Thus, more buried objects are detected in the ground than normal. Wavelet transform was applied to eliminate these reflections and overlaps.
It is provided that buried objects can be observed more clearly. Buried objects and ground surface were segmented by the Otsu thresholding method. Hough transformation was used to eliminate the soil surface.

In order to obtain the highest accuracy rate for buried object detection, three different features have been extracted from the segmented image. These are GLCM, RLM and ACF features. These features were given both directly as input to the classification algorithms and reduced in size using PCA and ICA. The main purpose of using PCA and ICA algorithms is to represent higher quality features from the segmented images by reducing the high dimensional features. When examined in Table 1, it showed a positive effect on the accuracy rates of feature reduction methods. Buried objects tried to be detected by classification process. Here, 23 classification algorithms are used to achieve the highest classification performance.

\section{Results}

Implementation results and performance results of proposed algorithms are described in this section. GprMax program, an electromagnetic simulator, was used to create scenarios for buried objects. It proposes an iterative solution based on the finitedifference time-domain numerical method to Maxwell equations both in space and time. In the simulation program, the properties of the transmission and acquisition system need to be adjusted. In GPR scenarios, dipole antenna is modeled and signal center frequency is $400 \mathrm{MHz}$. It also has a $400 \mathrm{MHz}$ bandwidth and a pulse length of $2.5 \mathrm{~ns}$. Three different soil types were used for the ground whose dimensions were fixed to $3 \times 4 \mathrm{~m}$. After these settings were completed, 180 GPR scenarios were created for the proposed method.

Noise reduction in GPR images was achieved with DWT. Otsu thresholding method and Hough transformation were applied respectively for segmentation of images. GLCM, RLM and ACF matrix were extracted for detection of rectangular, cylindrical and triangular objects. For each scenario, feature vectors consisting of statistical feature values were generated. These properties were normalized prior to classification process. Using LDA, QDA, KNN, ANN, SVM and DT, buried objects with different shapes are classified. These objects have cylindrical, rectangular and triangular shapes. 


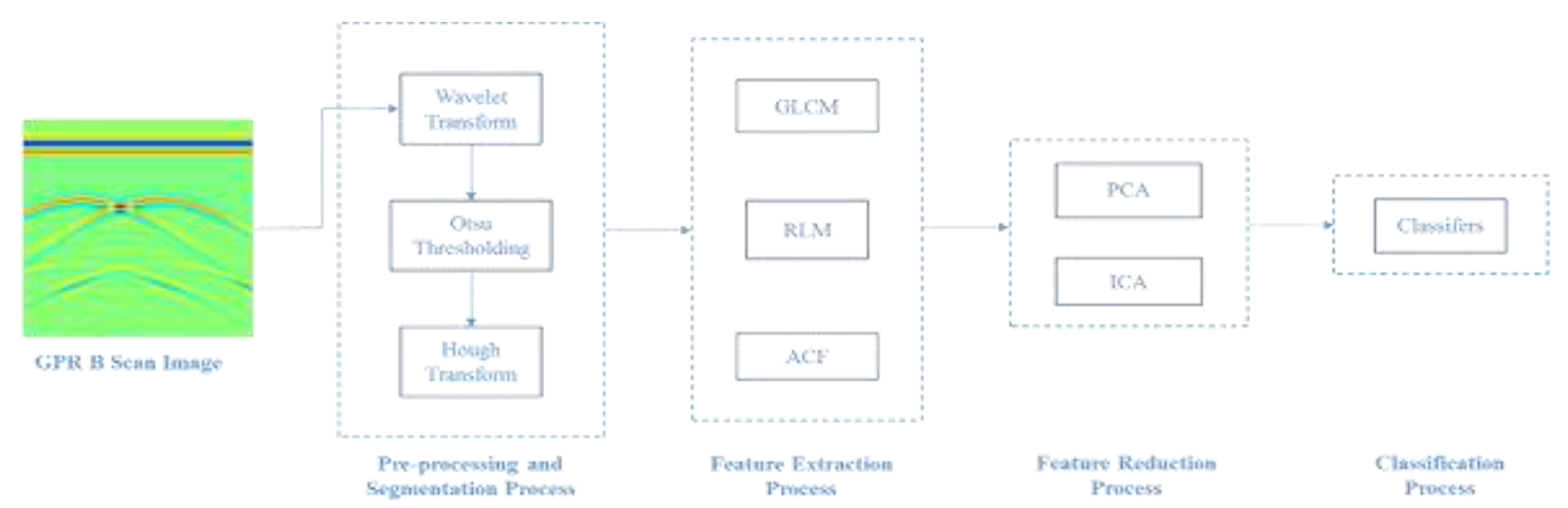

Fig. 3. Proposed Algorithm Procedure

As shown in Table 1, approximately 164 (91.7\%) scenarios could be detected correctly. In other words the shape of the different materials in the image was determined correctly. In GPR applications, maximum tolerance is $10 \mathrm{~cm}$ for object position. If this tolerance is not exceeded, it is assumed that the object has been correctly detected. Scenarios were created by considering three different grounds and five different material properties in the field of view. GPR images can cause buried objects that are not found in the scenario to be detected as a result of overlapping hyperbolas created by other buried objects.

Table 1 shows that Coarse KNN has the lowest classification accuracy rate of $21.4 \%$ with using
ACF features. The highest classification performance is Coarse Gaussian SVM of GLCM features where the feature matrix is reduced using PCA with $91.7 \%$. Complex Tree, Medium Tree and Simple Tree have the highest average classification accuracy with $75.8 \%$. Boosted Trees has the lowest accuracy with $35.58 \%$ in the classification. GLCM+PCA features have the highest average accuracy of $76.24 \%$. On the other hand, the lowest classification accuracy is ACF + PCA with $60.5 \%$. The average accuracy rates of GLCM, RLM and $\mathrm{ACF}$ in classification are $73.1 \%, 67.27 \%$ and $66.27 \%$ respectively. The average classification performance of all features is $68.88 \%$.

Table 1. Accuracies of Classification Algorithms

\begin{tabular}{|c|c|c|c|c|c|c|c|c|c|}
\hline Classifier & GLCM & GLCM+PCA & GLCM+ICA & RLM & $\mathrm{RLM}+\mathrm{PCA}$ & RLM+ICA & $\mathrm{ACF}$ & $\mathrm{ACF}+\mathrm{PCA}$ & $\mathrm{ACF}+\mathrm{ICA}$ \\
\hline Complex Tree & $86.1 \%$ & $88.9 \%$ & $81.7 \%$ & $91.7 \%$ & $66.7 \%$ & $71.7 \%$ & $66.7 \%$ & $61.1 \%$ & $67.8 \%$ \\
\hline Medium Tree & $86.1 \%$ & $88.9 \%$ & $81.7 \%$ & $91.7 \%$ & $66.7 \%$ & $71.7 \%$ & $66.7 \%$ & $61.1 \%$ & $67.8 \%$ \\
\hline Simple Tree & $86.1 \%$ & $88.9 \%$ & $81.7 \%$ & $91.7 \%$ & $66.7 \%$ & $71.7 \%$ & $66.7 \%$ & $61.1 \%$ & $67.8 \%$ \\
\hline LDA & $66.7 \%$ & $66.7 \%$ & $66.7 \%$ & $80.6 \%$ & $75.0 \%$ & $68.9 \%$ & $66.7 \%$ & $69.4 \%$ & $87.2 \%$ \\
\hline QDA & $77.8 \%$ & $80.6 \%$ & $77.8 \%$ & $77.8 \%$ & $77.8 \%$ & $74.4 \%$ & $58.3 \%$ & $80.6 \%$ & $81.7 \%$ \\
\hline Linear SVM & $88.9 \%$ & $86.1 \%$ & $77.8 \%$ & $83.3 \%$ & $72.2 \%$ & $68.9 \%$ & $66.7 \%$ & $55.6 \%$ & $84.4 \%$ \\
\hline Quadratic SVM & $83.3 \%$ & $80.6 \%$ & $86.1 \%$ & $80.6 \%$ & $72.2 \%$ & $71.4 \%$ & $63.9 \%$ & $69.4 \%$ & $73.3 \%$ \\
\hline Cubic SVM & $75.0 \%$ & $86.1 \%$ & $69.4 \%$ & $77.8 \%$ & $72.2 \%$ & $74.4 \%$ & $63.9 \%$ & $63.9 \%$ & $73.3 \%$ \\
\hline Fine Gaussian SVM & $69.4 \%$ & $86.1 \%$ & $86.1 \%$ & $30.6 \%$ & $33.3 \%$ & $66.1 \%$ & $80.6 \%$ & $72.2 \%$ & $67.8 \%$ \\
\hline Medium Gaussian SVM & $86.1 \%$ & $86.1 \%$ & $80.6 \%$ & $80.6 \%$ & $83.3 \%$ & $71.7 \%$ & $80.6 \%$ & $80.6 \%$ & $65.0 \%$ \\
\hline Coarse Gaussian SVM & $55.6 \%$ & $91.7 \%$ & $55.6 \%$ & $47.2 \%$ & $50.0 \%$ & $68.9 \%$ & $47.2 \%$ & $47.2 \%$ & $67.8 \%$ \\
\hline Fine KNN & $83.3 \%$ & $83.3 \%$ & $83.3 \%$ & $63.9 \%$ & $58.3 \%$ & $57.8 \%$ & $75.0 \%$ & $66.7 \%$ & $81.7 \%$ \\
\hline Medium KNN & $75.0 \%$ & $77.8 \%$ & $75.0 \%$ & $72.2 \%$ & $61.1 \%$ & $77.2 \%$ & $69.4 \%$ & $58.3 \%$ & $81.7 \%$ \\
\hline Coarse KNN & $27.8 \%$ & $27.8 \%$ & $27.8 \%$ & $27.8 \%$ & $67.8 \%$ & $57.8 \%$ & $21.4 \%$ & $27.8 \%$ & $67.8 \%$ \\
\hline Cosine KNN & $66.7 \%$ & $83.3 \%$ & $66.7 \%$ & $69.4 \%$ & $66.7 \%$ & $74.4 \%$ & $58.3 \%$ & $61.1 \%$ & $76.1 \%$ \\
\hline Cubic KNN & $66.7 \%$ & $77.8 \%$ & $75.0 \%$ & $44.4 \%$ & $58.3 \%$ & $82.8 \%$ & $55.6 \%$ & $61.1 \%$ & $81.7 \%$ \\
\hline Weighted KNN & $83.3 \%$ & $86.1 \%$ & $83.3 \%$ & $66.7 \%$ & $63.9 \%$ & $68.9 \%$ & $75.0 \%$ & $66.7 \%$ & $87.2 \%$ \\
\hline Boosted Trees & $27.8 \%$ & $27.8 \%$ & $27.8 \%$ & $27.8 \%$ & $27.8 \%$ & $57.8 \%$ & $27.8 \%$ & $27.8 \%$ & $67.8 \%$ \\
\hline Bagged Trees & $86.1 \%$ & $83.3 \%$ & $83.3 \%$ & $80.6 \%$ & $66.7 \%$ & $63.3 \%$ & $75.0 \%$ & $66.7 \%$ & $84.4 \%$ \\
\hline Subspace Discriminant & $88.9 \%$ & $69.4 \%$ & $55.6 \%$ & $80.6 \%$ & $77.8 \%$ & $66.1 \%$ & $63.9 \%$ & $63.9 \%$ & $87.2 \%$ \\
\hline Subspace KNN & $83.3 \%$ & $83.3 \%$ & $83.3 \%$ & $69.4 \%$ & $80.6 \%$ & $63.3 \%$ & $61.1 \%$ & $66.7 \%$ & $84.4 \%$ \\
\hline RUS Boosted Trees & $41.7 \%$ & $41.7 \%$ & $41.7 \%$ & $41.7 \%$ & $55.6 \%$ & $74.4 \%$ & $38.9 \%$ & $44.4 \%$ & $70.6 \%$ \\
\hline ANN & $77.7 \%$ & $81.4 \%$ & $73.3 \%$ & $73.3 \%$ & $73.3 \%$ & $73.3 \%$ & $88.9 \%$ & $58.3 \%$ & $62.2 \%$ \\
\hline
\end{tabular}




\section{Conclusion}

In this study, a comparative analysis system of GPR images is presented. The proposed system allows to determine the buried of embedded objects. After the pre-processing and segmentation stages, different types of features were extracted and classification was performed. It has been tried to obtain the highest accuracy detection rate among proposed classifiers. Despite the reflections and overlaps in GPR images, the experimental results show up to $91.7 \%$ detection performance. The experimental results show that the proposed system has a high accuracy rate in terms of estimating the object shape even if buried objects are close to each other. This performance also depends on the number of GPR images in order to properly train the classifier in the proposed system.

\section{References}

1. S. Shihab and W. Al-Nuaimy, "Radius estimation for cylindrical objects detected by ground penetrating radar," Subsurf. Sens. Technol. Appl., vol. 6, no. 2, pp. 151166, Apr. 2005.

2. L. Capineri, P. Grande, and J. A. G. Temple, "Advanced image-processing technique for real-time interpretation of ground-penetrating radar images," Int. J. Imaging Syst. Technol., vol. 9, no. 1, pp. 51-59, 1998

3. S. Caorsi and G. Cevini, "An electromagnetic approach based on neural networks for the GPR investigation of buried cylinders," IEEE Geosci. Remote Sens. Lett., vol. 2, no. 1, pp. 3-7, 2005.

4. A. Dolgiy, A. A. Dolgiy, and V. Zolotarev, "Estimation of subsurface pipe radius using ground penetrating radar data," in Proc. 12th Eur. Meeting Environ. Eng. Geophys., Helsinki, Finland, Sep. 4-6, 2006, pp. 1-5.

5. H. Brunzell, "Detection of shallowly buried objects using impulse radar," IEEE Trans. Geosci. Remote Sens., vol. 37, no. 2, pp. 875-886, Mar. 1999.

6. S. Delbò, P. Gamba, and D. Roccato, “A fuzzy shell clustering approach to recognize hyperbolic signatures in subsurface radar images," IEEE Trans. Geosci. Remote Sens., vol. 38, no. 3, pp. 1447-1451, May 2000.

7. P. Gamba and S. Lossani, "Neural detection of pipe signatures in ground penetrating radar images," IEEE Trans. Geosci. Remote Sens., vol. 38, no. 2, pp. 790-797, Mar. 2000.

8. W. Al-Nuaimy, Y. Huang, M. Nakhkash,M. T. C. Fang, V. T. Nguyen, and A. Eriksen, "Automatic detection of buried utilities and solid objects with GPR using neural networks and pattern recognition," J. Appl. Geophys., vol. 43, no. 2-4, pp. 157-165, Mar. 2000.

9. H. S. Youn and C. C. Chen, "Automatic GPR target detection and clutter reduction using neural network," in Proc. 9th Int. Conf. Ground Penetrating Radar, Santa Barbara, CA, 2002, vol. 4758, pp. 579582.

10. M. Rossini, "Detecting objects hidden in the subsoil by a mathematical method," Comput. Math. Appl., vol. 45, no. 1, pp. 299-307, Jan. 2003.

11. S. Shihab, W. Al-Nuaimy, and Y. Huang, "A comparison of segmentation techniques for target extraction in ground penetrating radar data," in Proc. 2nd Int. Workshop Advanced GPR, Delft, The Netherlands, 2003, pp. 95-100.

12. P. Gamba and V. Belotti, "Two fast buried pipe detection schemes in ground penetrating radar images," Int. J. Remote Sens., vol. 24, no. 12, pp. 2467-2484, Jan. 2003.

13. P. Falorni, L. Capineri, L. Masotti, and G. Pinelli, "3-D radar imaging of buried utilities by features estimation of hyperbolic diffraction patterns in radar scans," in Proc. 10th Int. Conf. Ground Penetrating Radar, Delft, The Netherlands, 2004, vol. 1, pp. 403-406.

14. A. Dell'Acqua, A. Sarti, S. Tubaro, and L. Zanzi, "Detection of linear objects in GPR data," Signal Process., vol. 84, no. 4, pp. 785-799, Apr. 2004.

15. A. C. Gurbuz and J. H. McClellan, "Iterative detection of linear objects in GPR and seismic images," in Proc. 4th IEEE Workshop Sensor Array Multichannel Signal Process, Waltham, MA, 2006, pp. 118-121.

16. G. Borgioli, L. Capineri, P. Falorni, S. Mattucci, and C. G. Windsor, "The detection of buried pipes from time-offlight radar data," IEEE Trans. Geosci. Remote Sens., vol. 46, no. 8, pp. 22542266, Aug. 2008.

17. E. Pasolli, F. Melgani, and M. Donelli, "Automatic Analysis of GPR Images: A Pattern-Recognition Approach," IEEE Transactions on Geoscience and Remote Sensing, vol. 47, no. 7, pp. 2206-2217, 2009. 
18. E. Pasolli, F. Melgani, and M. Donelli, "Automatic Analysis of GPR Images: A Pattern-Recognition Ap-proach," IEEE Transactions on Geoscience and Remote Sensing, vol. 47, no. 7, pp. 2206-2217, 2009.

19. R.O. Duda and P.E. Hart, "Use of the Hough Transformation to Detect Lines and Curves in Pictures", Comm. ACM. Vol. 15, no.1, pp.11-15, 1972.

20. H. Wechsler and J. Sklansky, "Automatic Detection of Ribs in Chest Radiographs", Pattern Recognition, vol. 9, pp. 21-30, 1977.

21. K.E.Forkheim, D.Scuse, H.Pasterkamp, A comparison of neural network models for wheeze detection, in: IEEE WESCANEX 95 Proceedings, 1, (New York, USA 1995), pp. 214-219.

22. M. M. Galloway, "Texture analysis using gray level run lengths," Computer Graphics Image Processing, vol. 4, pp. 172-179, 1975.

23. Wang, X., \& Paliwal, K. K. (2003). Feature extraction and dimensionality reduction algorithms and their applications in vowel recognition, Pergamon. The Journal of the Pattern Recognition Society, 36, 24292439.

24. M. Turk and A. Pentland, Eigenfaces for recognition, J Cogn Neurosci 3 (1991), 7186.

25. M.S. Bartlett, J.R. Movellan, and T.J. Sejnowski, Face recognition by independent component analysis, IEEE Trans Neural Networks 13 (2002), 14501464.

26. B. Draper, K. Baek, M.S. Bartlett, and J.R. Beveridge, Recognizing faces with PCA and ICA, Comput Vis Image Understand (Special Issue on Face Recognition) 91 (2003), 115-137.

27. L.-F. Chen, H.-Y. M. Liao, M.-T. Ko, J.-C. Lin, and G.-J. Yu, "A new LDA-based face recognition system which can solve the small sample size problem," Pattern Recognit., vol. 33, no. 10, pp. 1713-1726, 2000.

28. Srivastava Santosh, Gupta Maya R., Frigyik B'ela A., "Bayesian Quadratic Discriminant Analysis" Journal of Machine Learning Research 8 (2007) 1277-1305.

29. T.M. Cover and P.E. Hart. Nearest neighbor pattern classification. IEEE Transactions on Information Theory, 13(1):021-027, 1967.

30. M. Latourrette. Toward an ecplanatory similarity measure for nearest neighbor classification. In ECML-2000, pages 238245, London, UK, 2000.
31. R. Kizılaslan and B. Karlık, Combination neural networks forecasters for monthly natural gas consumption prediction, Neural Netw World, 19 (2) (2009), pp. 191- 199.

32. R.O. Duda, P.E. Hart and D.G. Stork, Pattern classification and scene analysis, Wiley, (New York 2001).

33. A. Kandaswamy, C. S. Kumar, R. P. Ramanathan, S. Jayaraman and N. Malmurugan, Neural classification of lung sounds using wavelet coefficients, Comput. Biol. Med., 34 (2004), pp. 523-537.

34. Gunn Steve R.,"Support Vector Machines For Classification and Regression", Technical report, University of Southampton, May 1998.

35. H. Liu, A. Gegov, and M. Cocea, Rule Based Systems for Big Data: A Machine Learning Approach. Switzerland: Springer, 2016.

36. Srivastava Santosh, Gupta Maya R., Frigyik B'ela A., "Bayesian Quadratic Discriminant Analysis" Journal of Machine Learning Research 8 (2007) 1277-1305. 